Review

\title{
Developments in Molecular Recognition and Sensing at Interfaces
}

\section{Katsuhiko Ariga*, Jonathan P. Hill and Hiroshi Endo}

Supermolecules Group, National Institute for Materials Science (NIMS), 1-1 Namiki, Tsukuba 305-0044, Japan

* Author to whom correspondence should be addressed; E-mail: ARIGA.Katsuhiko@nims.go.jp Received: 6 August 2007 / Accepted: 20 August 2007 / Published: 22 August 2007

\begin{abstract}
In biological systems, molecular recognition events occur mostly within interfacial environments such as at membrane surfaces, enzyme reaction sites, or at the interior of the DNA double helix. Investigation of molecular recognition at model interfaces provides great insights into biological phenomena. Molecular recognition at interfaces not only has relevance to biological systems but is also important for modern applications such as high sensitivity sensors. Selective binding of guest molecules in solution to host molecules located at solid surfaces is crucial for electronic or photonic detection of analyte substances. In response to these demands, molecular recognition at interfaces has been investigated extensively during the past two decades using Langmuir monolayers, selfassembled monolayers, and lipid assemblies as recognition media. In this review, advances of molecular recognition at interfaces are briefly summarized.
\end{abstract}

Keywords: molecular recognition, interface, air-water interface, Langmuir-Blodgett film, self-assembled monolayer, molecular assembly.

\section{Introduction}

Molecular recognition is one of the most important chemical events in biological systems and has been mimicked in supramolecular chemistry as, for example, artificial enzymes [1-3]. Most of the supramolecular systems for molecular recognition are composed of molecularly dispersed states in homogeneous solutions. However, this is quite apart from the situation seen in biological systems. Molecular recognition in living systems occurs mostly at interfacial environments such as membrane surfaces, enzyme reaction sites, or at the interior of the DNA double helix. 
Why have biological systems adopted interfacial environments for molecular recognition? A part of the answer was provided by pioneering work by Kunitake and co-workers [4] who compared binding efficiency between guanidinium and phosphate in three different environments (Figure 1). Binding constants of adenosine monophosphate (AMP) to guanidinium functionality in aqueous aggregates such as micelle or bilayer vesicles were evaluated at $10^{2}-10^{4} \mathrm{M}^{-1}$. These values are significantly larger than those between molecularly-dispersed guanidinium and phosphate in water $\left(1.4 \mathrm{M}^{-1}\right)$ [5]. Surprisingly, a large enhancement in binding constant was reported for binding of AMP to guanidinium groups when embedded at a water surface [6]. These results clearly indicate that molecular recognition can be achieved much more efficiently at an appropriate interface.



$\mathrm{K}=1.4 \mathrm{M}^{-1}$

(a) in Aqueous Solution

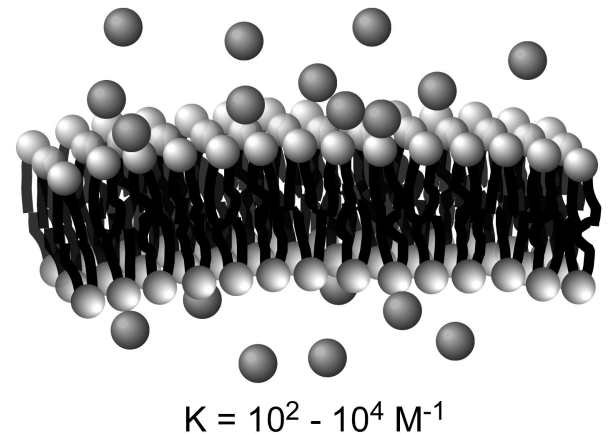

(b) on Surface of Miceles and Bilayers

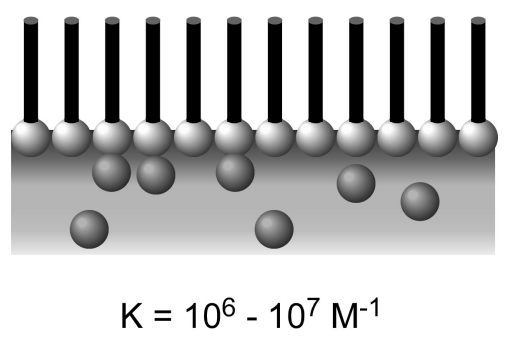

(c) at Air-Water Interface

Figure 1. Typical binding constants between phosphate and guanidinium for various aqueous media interfaces: (a) in aqueous solution; (b) on the surface of micelles and bilayers; (c) at the air-water interface. Reprinted with permission from [4] @1996, American Chemical Society.

In order to further understand these findings, Sakurai and co-workers considered theoretical aspects of molecular recognition at the air-water interface using a quantum chemical approach including reaction field calculations combined with AM1 molecular orbital methods [7-9]. Guanidinium host and phosphate guest were placed in various positions at a model interface consisting of a lipid layer (low dielectric, $\varepsilon=2$ ) and water phase (high dielectric, $\varepsilon=80$ ). The calculated binding energies depended significantly on the position of the binding site relative to the two-phase boundary. A large binding constant was obtained when the binding site was located in the lipid phase, while positioning the guanidinium deep in the water phase resulted in a very small binding energy. Even when the hydrogen bonding site was positioned in the water phase, the site is affected electronically by the low dielectric lipid layer, which strengthens intermolecular hydrogen bonding and electrostatic interactions. As a result, the binding constant increases significantly at the border between lipid and aqueous phases. These quantum chemical calculations suggested advantageous aspects of interfacial environments for efficient molecular recognition of substances in aqueous media.

Molecular recognition at interfaces not only has relevance to biological systems but also is important for modern applications such as high sensitivity sensors. Selective binding of guest 
molecules in solution to host molecules located at a solid surface is crucial for electronic and photonic detection of target substances. In response to these demands, molecular recognition at interfaces has been researched extensively during the past two decades using Langmuir monolayers, self-assembled monolayers, and lipid assemblies as recognition media. In this review, advances of molecular recognition at interfaces are briefly summarized.

\section{Molecular Recognition at the Air-Water Interface and Related Interfaces}

A molecular monolayer on a water surface provides an attractive medium for an aqueous guest to interact with host sites at the surface of the functionalized monolayer, while Langmuir monolayers can be also regarded as media appropriate for research on molecular recognition between membrane components [10,11]. Binding of aqueous ionic species to host monolayer surfaces is one of the most popular targets in this research area, as summarized in a review by Kruppa and Köning [12]. Okahata and co-workers investigated binding of calcium ions to phospholipid monolayers through the combined study of surface pressure $(\pi)$ - molecular area (A) isotherm measurement, electrochemical response, and quartz crystal microbalance (QCM) [13]. Ariga et al. reported selective detection of iodide in admixture with other halogen anions by using the change in aggregation mode within a monolayer of $\mathrm{N}$-confused tetraphenylporphyrin, which induces drastic variation in the absorption spectrum of the porphyrin [14]. Leblanc and co-workers demonstrated metal ion binding on mixed monolayers of peptide amphiphiles having GlyHisGly-related peptide sequence based on combinatorial surfacechemistry [15]. Monolayers can be transferred onto solid supports by using the Langmuir-Blodgett (LB) technique sometimes leading to useful sensing materials. Matsunaga and co-workers used an LB film composed of 4- $n$-dodecyl-6-(2-thiazolylazo)resorcinol for naked-eye detection of submicromolar levels of cadmium ions [16]. This probe membrane exhibited visual color transition, forming a series of reddish-orange to pinkish-purple complexes with cadmium, over a wide concentration range (0.04$44.5 \mu \mathrm{M})$.

Binding of neutral substances to Langmuir monolayers has been also extensively investigated, as seen in the rather ambiguous interaction with poly(vinylpyrrolidone) reported by Ariga et al. [17] and in the highly specific recognition of 1-phenylethylamine reported by Miyashita and co-workers [18]. Some of the most attractive guest molecules among neutral guests are the nucleic acid bases and related molecules. Pioneering work on molecular recognition of aqueous nucleic acid base was performed by Kitano and Ringsdorf who demonstrated changes in the $\pi$-A isotherm of an adeninefunctionalized monolayer upon addition of thymidine to the subphase [19]. Subsequently, direct evidence for hydrogen bond formation in a wide variety of systems including recognition of nucleotides, nucleic acid bases, and their analogues, was obtained by Kunitake and co-workers using analytical techniques such as Fourier-transform IR (FT-IR) and X-ray photoelectron spectroscopy (XPS) [20]. These target guests often have multiple binding sites so that translational freedom of molecules embedded at the air-water interface is advantageous for recognition of such guest molecules. In an example of a binary system, recognition of flavin mononucleotide (FMN) by mixed monolayers of dialkylmelamine and monoalkyl guanidinium has been demonstrated [21]. A ternary recognition system has been similarly designed where one flavin adenine dinucleotide (FAD) molecule has the potential to bind two guanidinium molecules at phosphate groups, one orotate molecule at adenine sites, 
and a diaminotriazine at an isoalloxiazine ring [22]. Formation of multi-point recognition sites in the mixed monolayer does not require great synthetic effort for the preparation of complicated covalentlylinked host compounds, which is usually obstacle for research of molecular recognition. Recently Vollhardt et al. reported on the recognition of thymine by 2,4-di(n-undecylamino)-6-amino-1,3,5triazine monolayers [23,24]. A combination of surface pressure studies with Brewster angle microscopy (BAM) imaging and grazing incidence X-ray diffraction (GIXD) measurements proved to be optimal for the characterization of the change in structure and phase behavior during the interfacial recognition process. Complementary hydrogen bonding of two thymine molecules by one melamine host molecule is deduced from the chemical structure of both components. Liang and co-workers also made considerable effort to analyse molecular recognition of nucleic acid base related molecules at the air-water interface $[25,26]$.

(a)

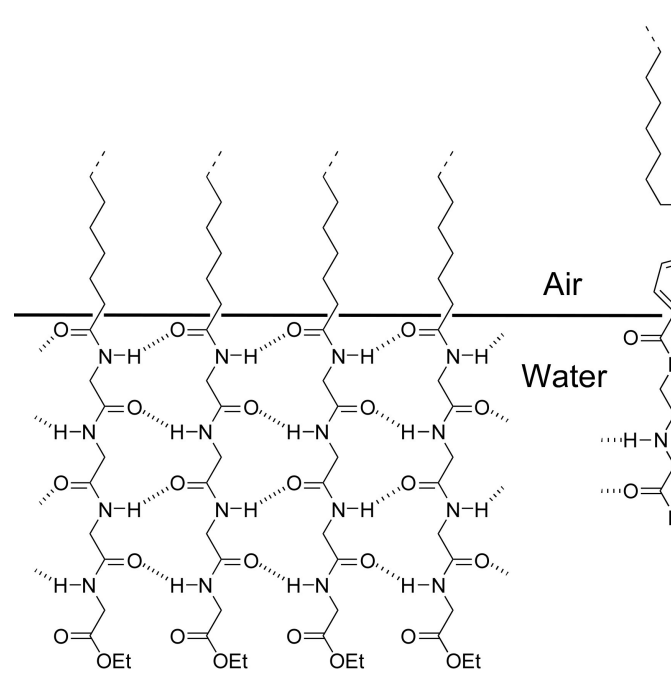

(c)



Figure 2. Various types of peptide-functionalized monolayer for molecular recognition of aqueous dipeptides (see text for details). Reprinted with permission from [30] (C)1996, American Chemical Society.

Some proteins, especially antibodies, are capable of performing highly selective molecular recognition tasks. Their recognition sites are composed of well-harmonized assemblies of peptide segments. Similarly assembled binding sites can be generated within monolayers at the air-water interface, as demonstrated by Kunitake and co-workers in their systematic research on recognition of aqueous peptides by peptide-amphiphile monolayers (Figure 2). An appropriate interval between host sites is the crucial point for successful recognition of the guest peptide molecules. Monoalkyl oligoglycine amphiphiles spread on water form strong hydrogen bonds within the monolayers and are not capable of accommodating external guest peptides contained in the subphase (a) [27]. Conversely, peptide binding together with guest selection was achieved in monolayers of dialkyl peptide amphiphiles in which the dialkylamine moiety was connected to the glycylglycinamide head group through a terephthaloyl unit, which endows an appropriate interval between the host peptide sites (b) [28]. Introduction of additional host functional groups that can interact with guest C-terminal or Nterminal should enhance the binding efficiency (c) [29-31]. Experimental results suggest that binding 
sites could be constructed spontaneously by an induced-fit mechanism and this similar concept has been used by other researchers. Leblanc and co-workers mimicked the binding site of acetylcholinesterase using a peptide-amphiphile with the PheTrpSerHisGlu segment and an octadecyloxy chain for selective recognition of paraoxon, sodium dihydrogen phosphate or 4nitrophenyl phosphate disodium [32]. Systematic investigation by $\pi$-A isotherm measurements revealed that the presence of Phe and Trp may be crucial for interaction with paraoxon. The same research group further investigated this recognition system using infrared reflection-absorption spectroscopy (IR-RAS) of the peptide-amphiphile Langmuir monolayer on subphases containing paraoxon [33]. It was revealed that the molecular recognition in this system involved $\pi-\pi$ interactions between the nitrobenzene group of paraoxon and aromatic groups in the peptide-amphiphile. Izhaky and Addadi also demonstrated the importance of spontaneous assembly of binding sites through stereoselective recognition by monoclonal antibodies of two-dimensional monolayers of cholesterol spread at the air-water interface [34]. Zadmard and Schrader reported nanomolar protein detection using an octadecanoic acid monolayer with a small amount (0.13 equiv) of one or two different calix[4]arene receptors, adorned with charged functional groups at their upper rims [35]. The recognition process requires a self-assembly of multiple calixarene units over the protein surface, which bind the protein in a cooperative fashion. Similarly, Schuster et al. investigated the enzymatic interplay of porcine pancreatic phospholipase $\mathrm{A}_{2}$ on a monolayer composed of dimyristoylphosphatidylethanolamine (DMPE) [36]. Oliviera and co-workers researched chitosan and phospholipid interactions using Langmuir and LB films as cell membrane models [37].

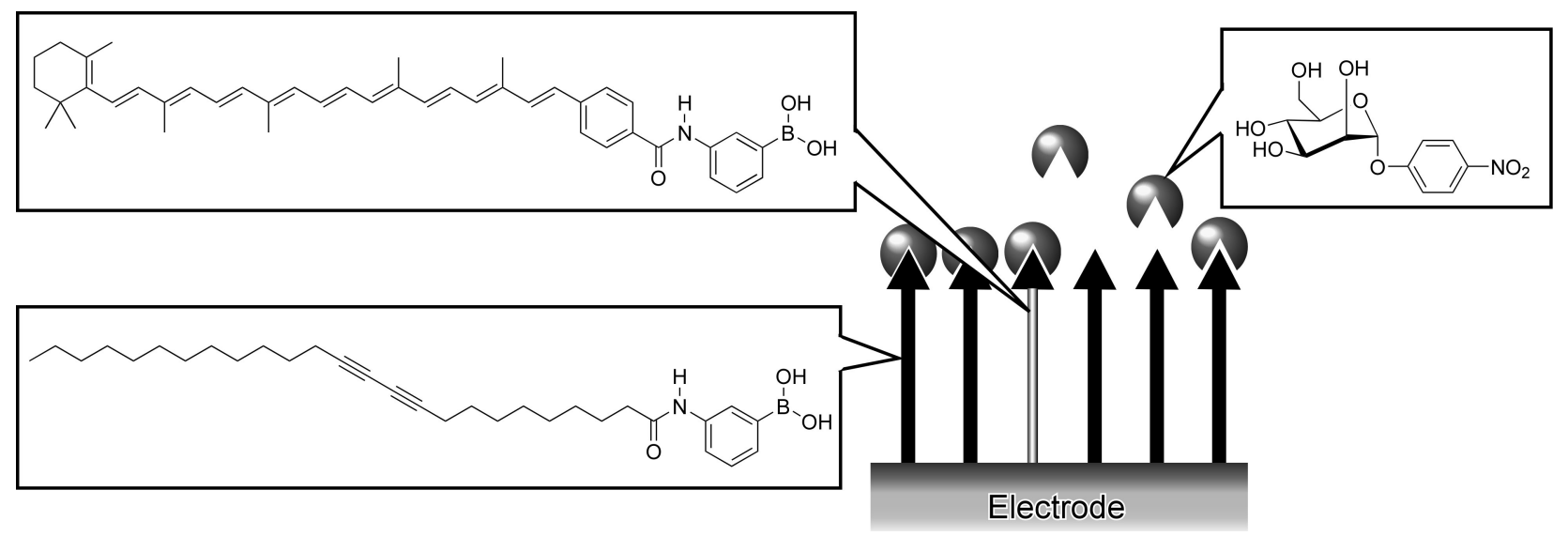

Figure 3. Recognition of sugar guest by boronic acid LB films. Reprinted with permission from [42] (C)2004, American Chemical Society.

Saccharide species are also important bioactive components. For example, recognition of saccharide is crucial in processes such as virus infection, and research on recognition of saccharide species has been recently investigated at the air-water interface [38]. Recognition of saccharides at the air-water interface was initiated in the late 1980s and 1990s and is exemplified in the cyclic resorcinol tetramer host reported by Kurihara et al. [39]. This was followed by several examples with synthetic hosts such as phenylboronic acid [40] or a macrocyclic sugar cluster. [41]. Recently, Miyahara and Kurihara reported rational design of LB films that are capable of electrochemical detection of redox-active saccharides (Figure 3) [42]. The boronic acid-functionalized, electroconductive LB film was fabricated 
by polymerization of a 1:10 mixture of a carotenoid type amphiphile and a polymerizable amphiphile. The selectivity of the electrode for sugar derivatives was achieved by attaching the molecular recognition site to the conjugated molecule. The conducting molecule with binding site, that is, the molecular wire with the connecting terminal, as designed in this study should open the possibility of designing well-defined complex molecular devices based on the available library of molecular assembly chemistry.

Binding of an aqueous guest to multiple amphiphiles in a monolayer should lead to formation of specific patterns in two-dimension [43,44]. The novel concept of two-dimensional molecular patterning was initiated by Kunitake and co-workers. In the first example, aqueous dicarboxylate can bind to two dialkylguanidinium molecules, and a spacer between the two carboxylates of the aqueous template affects the crystallinity of the guanidinium amphiphiles [45,46]. Monolayers transferred from aqueous oxalate (no methylene spacer) displayed a crystalline arc, whereas only an amorphous halo was observed for the monolayer from the pure water subphase. A rigid oxalate template bound the two guanidinium molecules and effectively weakened electrostatic repulsion among cationic guanidinium molecules through stoichiometric binding. When malonate $\left[\left(\mathrm{CH}_{2}\right)\right.$ spacer] or succinate $\left[\left(\mathrm{CH}_{2}\right)_{2}\right.$ spacer] was used as a template, the electron diffraction pattern could be assigned to a crystalline state, but the arc in the patterns broadened. A further increase in the spacer length [glutarate with $\left(\mathrm{CH}_{2}\right)_{3}$ spacer and adipate with $\left(\mathrm{CH}_{2}\right)_{4}$ spacer] again induced the amorphous phase of the monolayer. Melamine and barbituric acid (or related combinations) alternately bind through complementary hydrogen bonding, resulting in a molecular ribbon [47-49], which was kinetically analyzed by Vollhardt and co-workers using a sophisticated elemental set-up [50]. An AFM molecular image of the didodecylmelamine monolayer bound to the barbituric template could be easily observed, although the corresponding monolayer transferred from pure water was too fragile for AFM observation. An oblique array of methyl terminals was observed [51]. For molecular patterning with an aqueous template molecule having multiple binding sites, flavin adenine dinucleotide (FAD) was selected as an aqueous template and a recognition system with the guanidinium amphiphile and the orotate amphiphile was investigated (Figure 4) [52, 53]. AFM images of the formed complex transferred onto a mica surface showed the periodic brighter and darker portions that correspond to higher and lower regions of the monolayer surface, respectively. The height difference between the two peaks was estimated to be several angstroms. The AFM observation was similarly conducted on the corresponding monolayer transferred from pure water. The binding of FAD with the guanidinium/orotate mixed monolayer should dispose two functional units at the same level through binding to the template FAD molecule where the flavin unit in FAD may be buried under the phosphate group owing to the conformational flexibility of the FAD molecule, and resulting in a height difference between the two terminal methyl groups. 

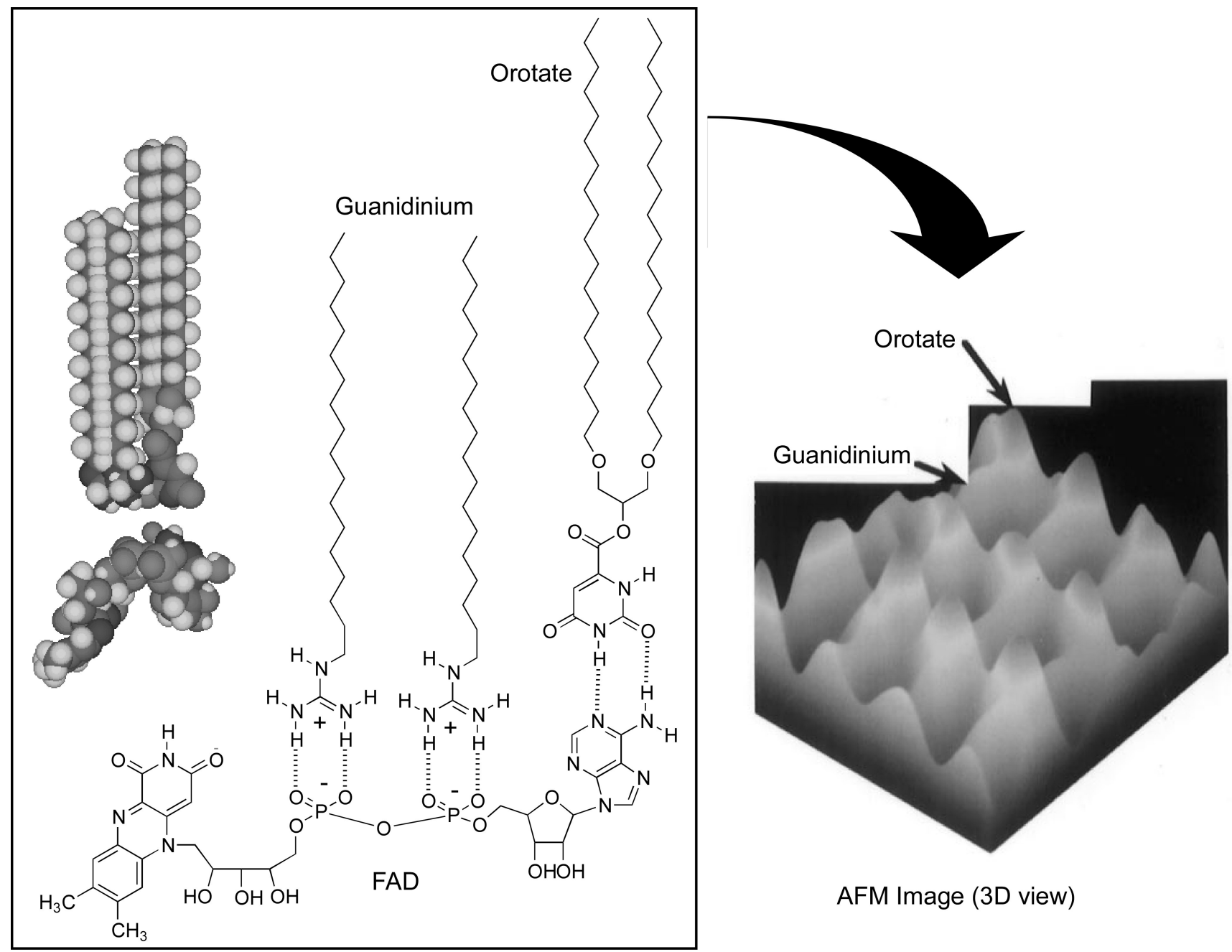

AFM Image (3D view)

Figure 4. Formation of a molecular pattern through molecular recognition of aqueous FAD. Reprinted with permission from [53] (C1997, American Chemical Society.

The air-water interface provides a flexible medium where conformational changes of amphiphiles are unobstructed. Therefore, regulation of molecular recognition based on conformational changes of host amphiphiles is one of the distinguishing characteristics of molecular recognition [54]. For example, Higuchi et al. reported $\mathrm{pH}$ control of molecular recognition at the air-water interface [55]. The poly(L-alanine) graft chains attached to amphiphilic polymer poly(allylamine) on water yielded sites which bind specifically with aqueous L-alanine rather than its $\mathrm{D}$ isomer. The specific binding of L-alanine to the monolayer was completely dependent on the structure of the monolayer, which can be regulated by variation of $\mathrm{pH}$. Ariga et al. realized mechanically controlled molecular recognition through dynamic formation of a cavity structure using host molecules, known as steroid cyclophanes [56-60]. The steroid cyclophane molecule used in this research contains a 1,6,20,25-tetraaza[6.1.6.1]paracyclophane cyclic core connected to four steroid moieties through a flexible L-lysine spacer with cholic acid (see Figure 5). In a monolayer at low pressures, it forms an open conformation in order to contact the hydrophilic face efficiently to the water phase. Compression of the monolayer forces the steroid cyclophane in the monolayer into a more compact conformation. As a result, the steroid cyclophane molecule adopts a cavity conformation at high pressures. Reversible binding (capture and release) of an aqueous fluorescent guest (6-( $p$-toluidino)naphthalene-2-sulfonate (TNS) was 
demonstrated by a repeated compression and expansion of the steroid cyclophane monolayer. According to repeated pressure application during the compression-expansion cycle, a periodic change in the fluorescence intensity was detected, because TNS emits fluorescence strongly in a hydrophobic host interior. The same research group applied a related system to aqueous molecular assemblies and realized modulation of emission wavelength [61]. The concept of dynamic function at the air-water interface has been recently similarly reported by other research groups. Diederich and co-workers proposed resorcin[4]arene cavitand-based molecular switches [62]. Resorcin[4]arene cavitands with four quinoxaline bridges are a family of macrocycles that adopt a contracted, vase-type conformation, capable of guest inclusion, whereas they switch to an expanded, kite-type conformation with a large flat surface under other conditions. This conformational switching was realized in Langmuir monolayers at the air-water interface. Stoddart, Ho, and co-workers demonstrated that redoxcontrollable molecular shuttles, in the shape of amphiphilic, bistable rotaxanes, switch mechanically under chemical stimulus even when contained in closely packed Langmuir films [63].



Figure 5. Dynamic molecular recognition by steroid cyclophane at the air-water interface. Reprinted with permission from [54] @2006, Royal Society of Chemistry.

More recently, Michinobu et al. reported control of enantioselectivity of amino acid recognition by dynamic motion of a polycholesteryl-substituted cyclen complex host molecule at the air-water interface [64]. The octacoordinate sodium complex has two possible quadruple helicate structures (see Figure 6). Helicity is influenced by the chirality of the side arms especially when ordered or aggregated at the supramolecular level. Application of lateral pressure should affect the helix structure and, consequently, the diastereomeric stability of complexes with guest molecules. The $K$ values of D- 
leucine are always greater than those of L-leucine, indicating that the cyclen monolayers have a stronger interaction with D-leucine. Conversely, the values of L-valine are smaller than those of Dvaline at low surface pressure but exceed them at $22-23 \mathrm{mN} \mathrm{m}^{-1}$. In other words, chiral recognition in the cyclen monolayers with valine changes from the D- to L-form upon compression. It is remarkable that such small difference in the chemical structure between leucine and valine can be distinguished by the dynamic process of monolayer formation.



Figure 6. Dynamic chiral recognition of amino acids by polycholesteryl-substituted cyclen complex at the air-water interface. Reprinted with permission from [64] @2006, American Chemical Society.

\section{Molecular Recognition at Other Interfaces}

Apart from the air-water interface, various interfacial environments provide media useful for molecular recognition and its related functions. In particular, self-assembled monolayers (SAM) are often used for sensor applications because the SAM structures facilitate contact with artificial devices such as electrodes and field effect transistors, as has been reviewed by Reinhoudt and co-workers [65]. Okahata and co-workers developed a siloxane-linked monolayer attached to a porous glass surface for permeation control [66-68]. They applied the same structure onto a tin dioxide electrode, where insertion of alkylalcohol into the alkylsiloxane monolayer could be electrochemically detected [69]. Geiger and co-workers reported use of an enhanced surface second harmonic generation (SHG) signal for detection of the toxic metal pollutant chromium(VI) at custom-made amino acid functionalized fused quartz/water interfaces [70]. Chromate adsorption isotherms recorded at $\mathrm{pH} 7$ were suggestive of an intramolecular chelation mechanism that would be important when four or more hydrogen-bonding moieties were displayed toward the incoming chromate. Credo et al. reported a method to manipulate conductance using molecular recognition at a SAM surface [71]. As illustrated in Figure 7, a binder molecule, diacyl 2,6-diaminopyridine decanethiolate (DAP, Figure 7A) was inserted into a background monolayer of decanethiolate on $\mathrm{Au}(111)$ using replacement lithography. Electroactive functionalization of the monolayer was then achieved through binding of the complementary ferrocene-terminated uracil 
to the binder molecule. Ferrocene function can be replaced by dodecyl uracil for erasing conductance. Current-voltage properties of the patterned region were monitored by using an STM tip. Noncovalent self-assembly provides a potential method to install and subsequently remove electroactive functionality in molecular electronics systems. Kitano and Taira used SAM structures of cyclodextrin derivatives for detection of bisphenol-type substances. They examined complexation of various kinds of bisphenols by a SAM of thiolated $\alpha$-cyclodextrin on a gold electrode by cyclic voltammetry using hydroquinone as a probe [72]. On the basis of the inhibitory effect of bisphenols on the inclusion of hydroquinone by the surface-confined cyclodextrin, the association constants of bisphenols with the immobilized $\alpha$-cyclodextrin were estimated. Use of $\beta$-cyclodextrin [73] and hexasodium calix [6]arene hexasulfonic acid [74] as host structures were also reported by the same research group. Willner and co-workers demonstrated photochemical imprinting of molecular recognition sites for phenoxynaphthacene quinone in SAM assembled on Au surfaces [75]. The primary step of their approach includes the assembly of the trans-phenoxynaphthacenequinone monolayer, followed by the rigidification of the monolayer with long chain alkanethiols generating a densely packed quinone monolayer. The second process involves the photoisomerization of the monolayer to the ana-quinone state, followed by the nucleophilic displacement of the quinone with butylamine. The binding of phenoxynaphthacenequinone to the imprinted recognition sites reveals selectivity, and structurally related substrates did not associate with the imprinted sites.

(a) Pattern

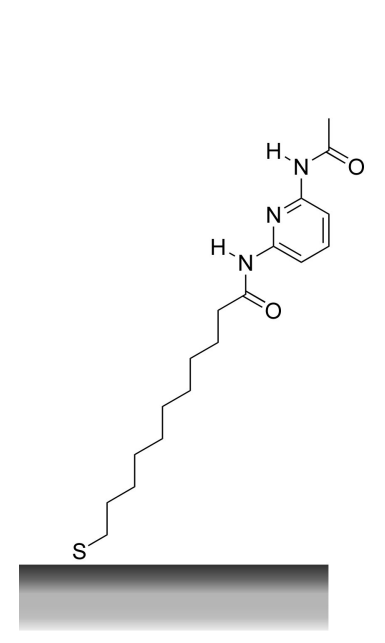

(b) Functionalize

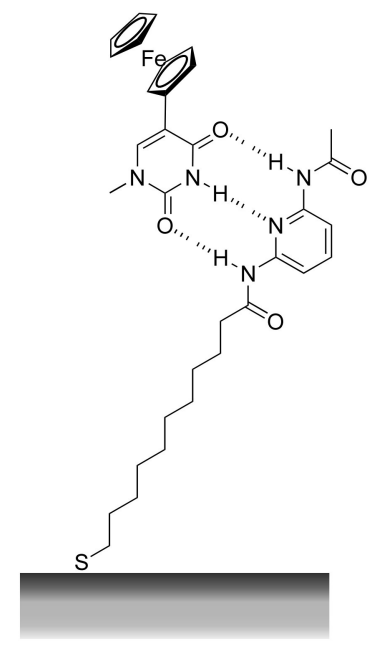

(c) Erase

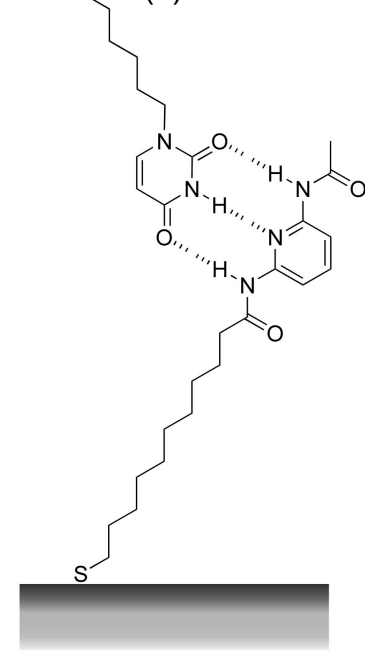

Figure 7. Functionalization and erasing at the surface of self-assembled monolayer. Reprinted with permission from [71] @2002, American Chemical Society.

Combining recognition events with appropriate analytical methods can yield sensor devices suitable for biological applications. Zeng, Wang, and co-workers demonstrated that an unlabeled carbohydrate mass sensor in combination with lectin-bacterial O-antigen recognition can be used for detection of high molecular weight bacterial targets with remarkably high sensitivity and enhanced specificity [76]. A functional mannose self-assembled monolayer in combination with lectin concanavalin A (Con A) was used as the molecular recognition element for detection of Escherichia coli W1485 using QCM as 
a transducer. Whitesides and co-workers reported the synthesis of bifunctional polyacrylamides containing pendant vancomycin and fluorescein groups, and the use of these polymers to direct antibodies against fluorescein to SAM presenting D-alanine-D-alanine groups [77]. Liu and Amiridis studied the interaction of avidin with biotin on functionalized quartz surfaces terminated with 3aminopropyltrimethoxysilane, 2,2'-(ethylenedioxy)bis(ethylenediamine), and fourth generation amineterminated polyamidoamine dendrimers, using the FTIR-RAS technique [78]. Advincula and co-workers reported nanomolar detection and specific recognition of pinacolyl methylphosphonate, a hydrolysis product and an analog of a relatively persistent class of toxic nerve agents [79]. In this system a modified polyamidoamine carbazole/ $\mathrm{Cu}^{2+}$ dendrimer, which is electrochemically cross-linked on a self-assembled monolayer (SAM) modified Au substrate, acted as an active sensing element for trapping the nerve agent analogs.

Other unique approaches using SAM structures have been reported. Bunker, Stoddart, and coworkers developed supramolecular machines using SAM structures in which molecular configurations can be reversibly programmed using electrochemical stimuli [80]. The proposed machines addressed the chemistry of substrate surfaces for integrated microfluidic systems. Interactions between the tethered tetracationic cyclophane host cyclobis(paraquat- $p$-phenylene) and dissolved $\pi$-electron-rich guest molecules, such as tetrathiafulvalene, were reversibly switched by oxidative electrochemistry. As reviewed by Rotello and co-workers [81], SAM structures can be prepared on size-controlled nanoparticles, providing scaffolds for sensing target molecules. Sanchez-Cortes and co-workers reported the use of 25,27-diethyl-dithiocarbamic-26,28-dihydroxy-p-tert-butylcalix[4]arene in the functionalization of $\mathrm{Ag}$ nanoparticles for pyrene detection by surface-enhanced Raman scattering (SERS) [82].

Immiscible liquids produce a dynamic interface such as the water-oil interface that have been also used as media for specific molecular recognition. Kitamura and co-workers investigated molecular recognition mediated by hydrogen-bonding interactions at a water- $\mathrm{CCl}_{4}$ interface by means of timeresolved total internal reflection (TIR) fluorescence spectroscopy [83]. In the presence of $\mathrm{N}, \mathrm{N}$ dioctadecyl-[1,3,5]triazine-2,4,6-triamine (DTT) in the $\mathrm{CCl}_{4}$ phase, the fluorescence decay profiles of riboflavin in aqueous phase were significantly modified, as compared with those observed in the absence of DTT, with the relevant amplitude varying with the concentration of DTT. Watarai and coworkers reported a molecular recognition system of the interfacial aggregation of monocationic palladium(II)-2-(5-bromo-2-pyridylazo)-5-diethylaminophenol complex with neutral diazine or purine bases at the toluene-water system [84]. The formation of interfacial aggregates of these complexes was investigated by centrifugal liquid membrane (CLM)/UV-Vis spectroscopy, CLM/resonance Raman spectroscopy and optical microscopy. The palladium(II)-2-(5-bromo-2-pyridylazo)-5diethylaminophenol complex formed interfacial aggregates preferentially with purine bases (adenine and guanine). Alkali metal ion recognition with [2-hydroxy-5-(4-nitrophenylazo)phenyl]-methyl-15crown-5 at the heptane-water interface was investigated by Teramae and co-workers, using in situ second harmonic generation (SHG) spectroscopy [85]. It was revealed experimentally that the $\mathrm{Na}^{+}$and $\mathrm{K}^{+}$complexes were flatter while the $\mathrm{Li}^{+}$complex exhibited a lift-up orientation at the heptane-water interface. 
Because of its relevance to biological systems, molecular recognition at the surface of aqueous lipid bilayers has also been investigated [86-91]. Sasaki et al. reported lead ion recognition by a crown ether functionalized lipid membrane [92]. The receptor-lipid with the crown ether at the head group and a pyrene fluorescent tag on the hydrophobic tail was synthesized and incorporated into bilayers of distearylphosphatidylcholine. The functionalized bilayer exhibited selective affinity for lead ions in aqueous buffered solution ( $\mathrm{pH}$ 7.4) and a fluorescence response that was linear over the concentration range $10^{-7}$ to $10^{-4} \mathrm{M}$ metal ions. Recognition and binding of lead ions at the membrane surface resulted in a rapid and prominent reorganization of the receptor-lipids in the membrane that was measurable at both the macro- and nanoscales. Removal of the lead ions, through the addition of EDTA, resulted in recovery of the original fluorescence and the reaggregation of structures in the membrane. Darcy, Ravoo, and co-workers synthesized amphiphilic cyclodextrins through 6-S-alkylation of the primary side and introduction of a poly(ethylene glycol) chain to the secondary side of $\alpha-, \beta-$, and $\gamma$ cyclodextrins, which formed nonionic bilayer vesicles in aqueous solution [93]. Molecular recognition of a hydrophobic anion (adamantane carboxylate) by the formed cyclodextrin vesicles was investigated by using capillary electrophoresis. The increase in electrophoretic mobility occurred when the hydrophobic anions bind to the nonionic cyclodextrin vesicles. Jelinek and co-workers proposed a molecular system in which interactions between antibodies and peptide epitopes displayed at a biomimetic membrane interface can be detected through induction of visible, rapid color transitions [94]. The colorimetric assembly consists of a phospholipid/polydiacetylene matrix anchoring a hydrophobic peptide displaying the epitope at its $\mathrm{N}$-terminus. The colorimetric transitions observed in the assembly, corresponding to perturbation of the polydiacetylene framework, are induced only upon recognition of the displayed epitope by its specific antibody present in the aqueous solution. This system could be utilized for studying antigen-antibody interactions and peptide-protein recognition, epitope mapping, and rapid screening of biological and chemical libraries.

Examples at other interfaces with and without matrices are briefly summarized below. Bohrer et al. fabricated chemiresistive gas sensors by deposition of $50 \mathrm{~nm}$ thick films of cobalt phthalocyanine and metal-free phthalocyanine on interdigitated gold electrodes via organic molecular beam epitaxy [95]. Belfort and co-workers prepared two-dimensional surface molecular imprinting method using water-inoil emulsion photo-polymerization on a microporous polypropylene substrate that was used to separate the bronchodilator, theophylline, from the mild stimulant, caffeine, both of similar chemical structure [96]. Kim et al. proposed a method for fabrication of patterned hydrogel microwells functionalized at their bases with antibodies to promote specific immobilization of lymphocytes [97]. Ma and coworkers reported an efficient silica coating process to prepare silica-coated gold nanorods [98]. The subsequent covalent bioconjugation of amino-functionalized gold nanorod films with goat anti-humanimmunoglobulin $\mathrm{G}$ (anti-h-IgG) was successfully employed for the colorimetric detection of h-IgG in a model reaction based on the specific binding affinity between the proteins. Govorov, Kotov, and coworkers prepared molecular spring assemblies of CdTe nanowires and Au nanoparticles [99], where the distance between the exciton and the plasmon can be reversibly varied. These systems were made protein-sensitive by incorporating antibodies in the molecular springs. Modulation of exciton-plasmon interactions could serve as a wavelength-based biodetection tool. 


\section{Future Perspectives}

Interfacial media provide a variety of possibilities for molecular recognition. At the air-water interface complicated binding sites can be constructed through self-assembly of rather simple molecular modules, where it is possible to generate well-defined recognition sites such as biomolecular receptors. Recently, Ariga et al. reported unusual shifts in dissociation constants of amino acid residues at the air-water interface that were accompanied by enzyme-like catalytic activities [100]. Thus, the airwater interface will be more important for developments in biomimetic chemistry in the future. On the other hand, solid surfaces should be useful for molecular sensor device preparation, for instance, in the preparation of sensing-site arrays which will be important for highly integrated molecular devices. Recent developments in control of molecular arrangements on two-dimensional solid surfaces [101103] should result in sensor arrays with ultrahigh spatial resolution. In addition, high-surface materials currently under development, such as mesoporous materials [104,105], should provide supports useful for efficient molecular recognition. A combination of advanced surface technologies and material chemistry with well-established molecular recognition sciences is crucial to the future development of this field.

\section{Acknowledgements}

The research described in this review was partially supported by Grant-in-Aid for Scientific Research on Priority Areas "Chemistry of Coordination Space" and a Grant-in-Aid for Science Research in a Priority Area "Super-Hierarchical Structures" from Ministry of Education, Science, Sports, and Culture, Japan, and Grants-in-Aid for Scientific Research (B) from Japan Society for the Promotion of Science.

\section{References and Notes}

1. Ariga, K.; Anslyn, E.V. Manipulating the stoichiometry and strength of phosphodiester binding to a bisguanidine cleft in DMSO/water solutions. J. Org. Chem. 1992, 57, 417-419.

2. Kneeland, D. M.; Ariga, K.; Lynch, V.M.; Huang, C.Y.; Anslyn, E.V. Bis(alkylguanidinium) receptors for phosphodiesters: effect of counterions, solvent mixtures, and cavity flexibility on complexation. J. Am. Chem. Soc. 1993, 115, 10042-10055.

3. Smith, J.; Ariga, K.; Anslyn, E.V. Enhanced imidazole-catalyzed RNA cleavage induced by a bisalkylguanidinium receptor. J. Am. Chem. Soc. 1993, 115, 362-364.

4. Onda, M.; Yoshihara, K.; Koyano, H.; Ariga, K.; Kunitake, T. Molecular recognition of nucleotides by the guanidinium unit at the surface of aqueous micelles and bilayers. A comparison of microscopic and macroscopic interfaces. J. Am. Chem. Soc. 1996, 118, 8524-8530.

5. Springs, B.; Haake, P. Equilibrium-constants for association of guanidinium and ammonium ions with oxyanions: effect of changing basicity of oxyanion. Bioorg. Chem. 1977, 6, 181-190.

6. Sasaki, D.Y.; Kurihara, K.; Kunitake, T. Specific, multiple point binding of ATP and AMP to a guanidinium-functionalized monolayer. J. Am. Chem. Soc. 1991, 113, 9685-9686. 
7. Sakurai, M.; Tamagawa, H.; Furuki, T.; Inoue, Y.; Ariga, K.; Kunitake, T. A theoretical interpretation of remarkable enhancement of intermolecular binding at the lipid-water interface. Chem. Lett. 1995, 1001-1002.

8. Sakurai, M.; Tamagawa, H.; Inoue, Y.; Ariga, K.; Kunitake, T. Theoretical study of intermolecular interaction at the lipid-water Interface. 1. Quantum chemical analysis using a reaction field theory. J. Phys. Chem. B 1997, 101, 4810-4816.

9. Tamagawa, H.; Sakurai, M.; Inoue, Y.; Ariga, K.; Kunitake, T. Theoretical study of intermolecular interaction at the lipid-water interface. 2. Analysis based on the Poisson-Boltzmann equation. $J$. Phys, Chem. B 1997, 101, 4817-4825.

10. Taneva, S.; Ariga, K.; Tagaki, W.; Okahata, Y. Association of amphiphilic cyclodextrins with dipalmitoylphosphatidylcholine in mixed insoluble monolayers at the air-water interface. $J$. Colloid Interface Sci. 1989, 131, 561-566.

11. Taneva, S.; Ariga, K.; Okahata, Y.; Tagaki, W. Association between amphiphilic cyclodextrins and cholesterol in mixed insoluble monolayers at the air-water interface. Langmuir 1989, 5, 111113.

12. Kruppa, M.; König, B. Reversible coordinative bonds in molecular recognition. Chem. Rev. 2006, 106, 3520-3560.

13. Ebara, Y.; Ebato, H.; Ariga, K.; Okahata, Y. Interactions of calcium ions with phospholipid membranes. Studies on $\pi$-A isotherms and electrochemical and quartz-crystal microbalance measurements. Langmuir 1994, 10, 2267-2271.

14. Ariga, K.; Kunitake, T.; Furuta, H. Specific binding of iodide ion to N-confused tetraphenylporphyrin (NC-TPP) at the air-water interface. J. Chem. Soc., Perkin Trans. 2 1996, 667-672.

15. Huo, Q.; Sui, G.; Zheng, Y.; Kele, P.; Leblanc, R.M.; Hasegawa, T.; Nishijo, J.; Umemura, J. Metal complexation with Langmuir monolayers of histidyl peptide lipids. Chem. Eur. J. 2001, 22, 4796-4804.

16. Prabhakaran, D.; Yuehong, M.; Nanjo, H.; Matsunaga, H. Naked-eye cadmium sensor: using chromoionophore arrays of Langmuir-Blodgett molecular assemblies. Anal. Chem. 2007, 79,40564065 .

17. Ariga, K.; Shin, J.S.; Kunitake, T. Interaction of lipid monolayers with aqueous neutral polymers and the consequent monolayer stabilization and improved Langmuir-Blodgett transfer. J. Colloid Interface Sci. 1995, 170, 440-448.

18. Qian P.; Matsuda, M. Miyashita, T. Chiral molecular recognition in polymer Langmuir-Blodgett films containing axially chiral binaphthyl groups. J. Am. Chem. Soc. 1993, 115, 5624-5628.

19. Kitano H.; Ringsdorf, H. Surface behaviors of nucleic acid base-containing lipids in monolayer and bilayer systems. Bull. Chem. Soc. Jpn. 1985, 58, 2826-2828.

20. Ariga, K.; Kunitake, T. Molecular recognition at air-water and related interfaces: complementary hydrogen bonding and multisite interaction. Acc. Chem. Res. 1998, 31, 371-378.

21. Ariga, K.; Kamino, A.; Koyano, H.; Kunitake, T. Recognition of aqueous flavin mononucleotide on the surface of binary monolayers of guanidinium and melamine amphiphiles. J. Mater. Chem. 1997, 7, 1155-1161. 
22. Taguchi, K.; Ariga, K.; Kunitake, T. Multi-site recognition of flavin adenine dinucleotide by mixed monolayers on water. Chem. Lett. 1995, 701-702.

23. Vollhardt, D.; Liu, F.; Rudert, R.; He, W. Interfacial molecular recognition of dissolved thymine by medium chain dialkyl melamine-type monolayers. J. Phys. Chem. B 2005, 109, 10849-10857

24. Vollhardt, D.; Liu, F.; Rudert, R. Molecular recognition of dissolved pyrimidine derivatives by a dialkyl melamine-type monolayer. ChemPhysChem 2005, 6, 1246-1250.

25. Miao, W.; Du, X.; Liang, Y. Molecular recognition of 1-(2-octadecyloxycarbonylethyl)cytosine monolayers to guanosine at the air-water interface investigated by infrared reflection-absorption spectroscopy. J. Phys. Chem. B 2003, 107, 13636-13642.

26. Wang Y., Du X., Miao, W.; Liang, Y. Molecular recognition of cytosine- and guaninefunctionalized nucleolipids in the mixed monolayers at the air-water interface and LangmuirBlodgett films. J. Phys. Chem. B 2006, 110, 4914-4923.

27. Cha, X.; Ariga, K.; Kunitake, T. Inter-peptide hydrogen bonding in monolayers of oligoglycine amphiphiles. Bull. Chem. Soc. Jpn. 1996, 69, 163-168.

28. Cha, X.; Ariga, K.; Onda, M.; Kunitake, T. Molecular recognition of aqueous dipeptides by noncovalently aligned oligoglycine units at the air/water interface. J. Am. Chem. Soc. 1995, 117, 11833-11838.

29. Cha, X.; Ariga, K.; Kunitake, T. Multi-site binding of aqueous dipeptides by mixed monolayers at the air-water interface. Chem. Lett. 1996, 73-74.

30. Cha, X.; Ariga, K.; Kunitake, T. Molecular recognition of aqueous dipeptides at multiple hydrogen-bonding sites of mixed peptide monolayers. J. Am. Chem. Soc. 1996, 118, 9545-9551.

31. Ariga, K.; Kamino, A.; Cha, X.; Kunitake, T. Multisite recognition of aqueous dipeptides by oligoglycine arrays mixed with guanidinium and other receptor units at the air-water interface. Langmuir 1999, 15, 3875-3885.

32. Wang C., Li, C.; Ji, X.; Orbulescu, J.; Xu, J.; Leblanc, R.M. Peptidolipid as binding site of acetylcholinesterase: molecular recognition of paraoxon in Langmuir films. Langmuir 2006, 22, 2200-2204.

33. Wang, C.; Zheng, J.; Oliveira, Jr.O.N.; Leblanc, R.M. Nature of the interaction between a peptidolipid Langmuir monolayer and paraoxon in the subphase. J. Phys. Chem. C 2007, 111, 7826-7833.

34. Izhaky, D; Addadi, L. Stereoselective interactions of a specialized antibody with cholesterol and epicholesterol monolayers. Chem. Eur. J. 2000, 6, 869-874.

35. Zadmard, R.; Schrader, T. Nanomolar protein sensing with embedded receptor molecules. J. Am. Chem. Soc. 2005, 127, 904-915.

36. Schuster, B.; Gufler, P.C.; Pum, D.; Sleytr, U.B. Interplay of phospholipase A2 with S-layersupported lipid monolayers. Langmuir 2003, 19, 3393-3397.

37. Pavinatto, F.J.; Caseli, L.; Pavinatto, A.; dos Santos, Jr., D.S.; Nobre, T.M.; Zaniquelli, M.E.D.; Silva, H.S.; Miranda, P.B.; de Oliveira, Jr.O.N. Probing chitosan and phospholipid interactions using Langmuir and Langmuir-Blodgett films as cell membrane models. Langmuir 2007, 23, 7666-7671. 
38. Matsuura, K.; Kitakouji, H.; Sawada, N.; Ishida, H.; Kiso, M.; Kitajima, K.; Kobayashi, K. A quantitative estimation of carbohydrate-carbohydrate interaction using clustered oligosaccharides of glycolipid monolayers and of artificial glycoconjugate polymers by surface plasmon resonance. J. Am. Chem. Soc. 2000, 122, 7406-7407.

39. Kurihara, K.; Ohto, K.; Tanaka, Y.; Aoyama, Y.; Kunitake, T. Molecular recognition of sugars by monolayers of resorcinol-dodecanal cyclotetramer. J. Am. Chem. Soc. 1991, 113, 444-450.

40. Ludwig, R.; Ariga, K.; Shinkai, S. Sensitive detection of saccharides by an amphiphilic phenylboronic acid at the air-water interface in the presence of quaternized amines. Chem. Lett. 1993, 1413-1416.

41. Ariga, K.; Isoyama, K.; Hayashida, O.; Aoyama, Y.; Okahata, Y. A QCM study on adsorption of macrocyclic sugar-cluster to variously-functionalized monolayers. Chem. Lett. 1998, 1007-1008.

42. Miyahara, T.; Kurihara, K. Electroconductive Langmuir-Blodgett films containing a carotenoid amphiphile for sugar recognition. J. Am. Chem. Soc. 2004, 126, 5684-5685.

43. Ariga, K. Template-assisted nano-patterning from submicron-scale to submolecular-level. $J$. Nanosci. Nanotechnol. 2004, 4, 23-34.

44. Ariga, K.; Nakanishi, T.; Takagi, N.; Tanaka, R.; Kikuchi, J. Two-dimensional molecular patterns and their dynamic functions: molecular recognition of aqueous guest by mixed monolayer of alkyl cyclophane and amphiphilic guanidinium. Colloid Surf. A-Physicochem. Eng. Asp. 2006, 284, 499-504.

45. Kamino, A.; Koyano, H.; Ariga, K.; Kunitake, T. Control of the molecular packing in guanidinium monolayers through binding with aqueous polycarboxylates. Bull. Chem. Soc. Jpn. 1996, 69, 3619-3631.

46. Oishi, Y.; Kato, T.; Kuramori, M.; Suehiro, K.; Ariga, K.; Kamino, A.; Koyano, H.; Kunitake, T. Control of molecular ordering in guanidinium-functionalized monolayer by carboxylate template molecules. Chem. Commun. 1997, 1357-1358.

47. Marchi-Artzner, V.; Artzner, F.; Karthaus, O.; Shimomura, M.; Ariga, K.; Kunitake, T.; Lehn, J.M. Molecular recognition between 2,4,6-triaminopyrimidine lipid monolayers and complementary barbituric molecules at the air/water interface: effects of hydrophilic spacer, ionic strength, and pH. Langmuir 1998, 14 5164-5171.

48. Koyano, H.; Bissel, P.; Yoshihara, K.; Ariga, K.; Kunitake, T. Syntheses and interfacial hydrogenbonded network of hexaalkyl tris(melamine) amphiphiles. Langmuir 1997, 13, 5426-5432.

49. Koyano, H.; Bissel, P.; Yoshihara, K.; Ariga, K.; Kunitake, T. Effect of melamine-amphiphile structure on the extent of two-dimensional hydrogen-bonded networks incorporating barbituric acid. Chem. Eur. J. 1997, 3, 1077-1082.

50. Kovalchuk, N.M.; Vollhardt, D.; Fainerman, V.B.; Aksenenko, E.V. Recognition and dissociation kinetics in the interfacial molecular recognition of barbituric acid by amphiphilic melamine-type monolayers. J. Phys. Chem. B 2007, 111, 8283-8289.

51. Koyano, H.; Yoshihara, K.; Ariga, K.; Kunitake, T.; Oishi, Y.; Kawano, O.; Kuramori, M.; Suehiro, K. Atomic force microscopic observation of a dialkylmelamine monolayer on barbituric acid. Chem. Commun. 1996, 1769-1770. 
52. Oishi, Y.; Torii, Y.; Kuramori, M.; Suehiro, K.; Ariga, K.; Taguchi, K.; Kamino, A.; Kunitake, T. Two-dimensional molecular patterning through molecular recognition. Chem. Lett. 1996, 411-412.

53. Oishi, Y.; Torii, Y.; Kato, T.; Kuramori, M.; Suehiro, K.; Ariga, K.; Taguchi, K.; Kamino, A.; Koyano, H.; Kunitake, T. Molecular patterning of a guanidinium/orotate mixed monolayer through molecular recognition with flavin adenine dinucleotide. Langmuir 1997, 13, 519-524.

54. Ariga, K.; Nakanishi, T.; Hill, J.P. A paradigm shift in the field of molecular recognition at the air-water interface: from static to dynamic. Soft Matter 2006, 2, 465-477.

55. Higuchi, M.; Wright, J.P.; Taguchi, K.; Kinoshita, T. Structure and molecular recognition properties of a poly(allylamine) monolayer containing poly(L-alanine) graft chains. Langmuir 2000, 16, 7061-7065.

56. Ariga, K.; Terasaka, Y.; Sakai, D.; Tsuji, H.; Kikuchi, J. Piezoluminescence based on molecular recognition by dynamic cavity array of steroid cyclophanes at the air-water interface. J. Am. Chem. Soc. 2000, 122, 7835-7836.

57. Ariga, K.; Tanaka, R.; Takagi, N.; Kikuchi, J. Molecular recognition by cyclophane/guanidinium supramolecular receptor embedded at the air-water interface. Supramol. Chem. 2003, 15, 87-94.

58. Ariga, K.; Nakanishi, T.; Terasaka, Y.; Tsuji, H.; Sakai, D.; Kukuchi, J. Piezoluminescence at the air-water interface through dynamic molecular recognition driven by lateral pressure application. Langmuir 2005, 21, 976-981.

59. Ariga, K.; Nakanishi, T.; Hill, J.P.; Terasaka, Y.; Sakai, D.; Kikuchi, J. Effect of guest capture modes on molecular recognition by a dynamic cavity array at the air-water interface: soft vs. tight and fast vs. slow. Soft Matter 2005, 1, 132-137.

60. Ariga, K.; Nakanishi, T.; Terasaka, Y.; Kikuchi, J. Catching a molecule at the air-water interface: dynamic pore array for molecular recognition. J. Porous Mater. 2006, 13, 427-430.

61. Ariga, K.; Sakai, D.; Terasaka, Y.; Tsuji, H.; Kikuchi, J. Information conversion on molecular assemblies containing steroid cyclophanes. Thin Solid Films 2001, 393, 291-297.

62. Azov, V.A.; Beeby, A.; Cacciarini, M.; Cheetham, A.G.; Diederich, F.; Frei, M.; Gimzewski, J.K.; Gramlich, V.; Hecht, B.; Jaun, B.; Latychevskaia, T.; Lieb, A.; Lill, Y.; Marotti, F.; Schlegel, A.; Schlittler, R.R.; Skinner, P.J.; Seiler, P.; Yamakoshi, Y. Resorcin[4]arene cavitand-based molecular switches. Adv. Funct. Mater. 2006, 16, 147-156.

63. Huang, T.J.; Tseng, H.R.; Sha, L.; Lu, W.; Brough, B.; Flood, A.H.; Yu, B.-D.; Celestre, P.C.; Chang, J.P.; Stoddart, J.F.; Ho, C.M. Mechanical shuttling of linear motor-molecules in condensed phases on solid substrates. Nano Lett. 2004, 4, 2065-2071.

64. Michinobu, T.; Shinoda, S.; Nakanishi, T.; Hill, J.P.; Fujii, K.; Player, T.N.; Tsukube, H.; Ariga, $\mathrm{K}$. Mechanical control of enantioselectivity of amino acid recognition by cholesterol-armed cyclen monolayer at the air-water interface. J. Am. Chem. Soc. 2006, 128, 14478-14479.

65. Flink, S.; van Veggel, F.C.J.M.; Reinhoudt, D.N. Sensor functionalities in self-assembled monolayers. Adv. Mater. 2000, 12, 1315-1328.

66. Okahata, Y.; Ariga, K.; Nakahara, H.; Fukuda, K. Permeation control by a phase transition of the dialkylsilane monolayer immobilized on a porous glass plate. J. Chem. Soc., Chem. Commun. 1986, 1069-1071. 
67. Okahata, Y.; Ariga, K.; Shimizu, O. Porous glass plate immobilized with the adsorbed monolayer of dialkylsilane amphiphiles. Permeation control by a phase transition of the adsorbed monolayer. Langmuir 1986, 2, 538-540.

68. Ariga, K.; Okahata, Y. Polymerized monolayers of single-, double-, and triple-chain silane amphiphiles and permeation control through the monolayer-immobilized porous glass plate in an aqueous solution. J. Am. Chem. Soc. 1989, 111, 5618-5622.

69. Okahata, Y.; Yokobori, M.; Ebara, Y.; Ebato, H.; Ariga, K. Electrochemical properties of covalently bonded silane amphiphile monolayers on a tin dioxide electrode. Langmuir 1990, 6, 1148-1153.

70. Gibbs-Davis, J.M.; Hayes, P.L.; Scheidt, K.A.; Geiger, F. M. Anion chelation by amido acid functionalized fused quartz/water interfaces studied by nonlinear optics. J. Am. Chem. Soc. 2007, 129, 7175-7184.

71. Credo, G.M.; Boal, A.K.; Das, K.; Galow, T.H.; Rotello, V.M.; Feldheim, D.L.; Gorman, C.B. Supramolecular assembly on surfaces: manipulating conductance in noncovalently modified mesoscale structures. J. Am. Chem. Soc. 2002, 124, 9036-9037.

72. Kitano, H.; Taira, Y. Inclusion of bisphenols by a self-assembled monolayer of thiolated cyclodextrin on a gold electrode. Langmuir 2002, 18, 5835-5840.

73. Endo, H.; Nakaji-Hirabayashi, T.; Morokoshi, S.; Gemmei-Ide, M.; Kitano, H. Orientational effect of surface-confined cyclodextrin on the inclusion of bisphenols Langmuir 2005, 21, 1314-1321.

74. Nakaji-Hirabayashi, T.; Endo, H.; Kawasaki, H,; Gemmei-Ide, M.; Kitano, H. Inclusion of bisphenols by a self-assembled monolayer of thiolated calix[6]arene on a gold surface. Environ. Sci. Technol. 2005, 39, 5414-5420.

75. Lahav, M.; Katz, E.; Willner, I. Photochemical imprint of molecular recognition sites in twodimensional monolayers assembled on Au electrodes: effects of the monolayer structures on the binding affinities and association kinetics to the imprinted interfaces. Langmuir 2001, 17, 73877395.

76. Shen, Z.; Huang, M.; Xiao, C.; Zhang, Y.; Zeng, X.; Wang, P.G. Nonlabeled quartz crystal microbalance biosensor for bacterial detection using carbohydrate and lectin recognitions. Anal. Chem. 2007, 79, 2312-2319.

77. Metallo, S. J.; Kane, R.S.; Holmlin, R.E.; Whitesides, G.M. Using bifunctional polymers presenting Vancomycin and fluorescein groups to direct anti-fluorescein antibodies to selfassembled monolayers presenting D-alanine-D-alanine groups. J. Am. Chem. Soc. 2003, 125, 4534-4540.

78. Liu Z; Amiridis, M.D. Quantitative FT-IRRAS spectroscopic studies of the interaction of avidin with biotin on functionalized quartz surfaces. J. Phys. Chem. B 2005, 109, 16866-16872.

79. Taranekar, P.; Baba, A.; Park, J.Y.; Fulghum, T.M.; Advincula, R. Dendrimer precursors for nanomolar and picomolar real-time surface plasmon resonance/potentiometric chemical nerve agent sensing using electrochemically crosslinked ultrathin films. Adv. Funct. Mater. 2006, 16, 2000-2007. 
80. Bunker, B.C.; Huber, D.L.; Kushmerick, J.G.; Dunbar, T.; Kelly, M.; Matzke, C.; Cao, J.; Jeppesen, J.O.; Perkins, J.; Flood, A.H.; Stoddart, J.F. Switching surface chemistry with supramolecular machines. Langmuir 2007, 23, 31-34.

81. You, C.-C.; Verma, A.; Rotello, V.M. Engineering the nanoparticle-biomacromolecule interface. Soft Matter 2006, 2, 190-204.

82. Guerrini, L.; Garcia-Ramos, J.V.; Domingo, C.; Sanchez-Cortes, S. Functionalization of Ag nanoparticles with dithiocarbamate calix[4]arene As an effective supramolecular host for the surface-enhanced Raman scattering detection of polycyclic aromatic hydrocarbons. Langmuir 2006, 22, 10924-10926

83. Ishizaka, S.; Kinoshita, S.; Nishijima, Y.; Kitamura, N. Direct observation of molecular recognition mediated by triple hydrogen bonds at a water/oil interface: time-resolved total internal reflection fluorometry study. Anal. Chem. 2003, 75, 6035-6042

84. Ohashi, A.; Tsukahara, S.; Watarai, H. Molecular recognition of diazine isomers and purine bases by the aggregation of palladium(II)-pyridylazo complex at the toluene/water interface. Langmuir 2003, 19, 4645-4651.

85. Nochi, K.; Yamaguchi, A.; Hayashita, T.; Uchida, T.; Teramae, N. Direct observation of alkali metal ion recognition processes at the heptane/water interface by second harmonic generation spectroscopy. J. Phys. Chem. B 2002, 106, 9906-9911.

86. Paleos, C.M.; Tsiourvas, D. Molecular recognition of organized assemblies via hydrogen bonding in aqueous media. Adv. Mater. 1997, 9, 695-710.

87. Kikuchi, J.; Ariga, K.; Miyazaki, T.; Ikeda, K. An artificial signal transduction system. Control of lactate dehydrogenase activity performed by an artificial cell-surface receptor. Chem. Lett. 1999, 253-254.

88. Kikuchi, J.; Ariga, K.; Ikeda, K. Signal transduction mediated by artificial cell-surface receptors: activation of lactate dehydrogenase triggered by molecular recognition and phase reorganization of bile acid derivatives embedded in a synthetic bilayer membrane. Chem. Commun. 1999, 547-548.

89. Kikuchi, J..; Ariga, K.; Sasaki, Y.; Ikeda, K. Control of enzymic activity by artificial cell-surface receptors. J. Mol. Catal. B-Enzym. 2001, 11, 977-984.

90. Fukuda, K.; Sasaki, Y.; Ariga, K.; Kikuchi, J. Dynamic behavior of a transmembrane molecular switch as an artificial cell-surface receptor. J. Mol. Catal. B-Enzym. 2001, 11, 971-976.

91. Pantos, A.; Tsiourvas, D.; Nounesis, G.; Paleos, C.M. Interaction of functional dendrimers with multilamellar liposomes: design of a model system for studying drug delivery. Langmuir 2005, 21, $7483-7490$.

92. Sasaki, D.Y.; Waggoner, T.A.; Last, J.A.; Alam, T.M. Crown ether functionalized lipid membranes: lead ion recognition and molecular reorganization. Langmuir 2002, 18, 3714-3721.

93. Falvey, P.; Lim, C.W.; Darcy, R.; Revermann, T.; Karst, U.; Giesbers, M.; Marcelis, A.T.M.; Lazar, A.; Coleman, A.W.; Reinhoudt, D.N.; Jan Ravoo, B.J. Bilayer vesicles of amphiphilic cyclodextrins: host membranes that recognize guest molecules. Chem. Eur. J. 2005, 11, 1171 1180 .

94. Kolusheva, S.; Kafri, R.; Katz, M.; Jelinek, R. Rapid colorimetric detection of antibody-epitope recognition at a biomimetic membrane interface. J. Am. Chem. Soc. 2001, 123, 417-422. 
95. Bohrer, F.I.; Sharoni, A.; Colesniuc, C.; Park, J.; Schuller, I.K.; Kummel, A.C.; Trogler, W.C. Gas sensing mechanism in chemiresistive cobalt and metal-free phthalocyanine thin films. $J . A m$. Chem. Soc. 2007, 129, 5640-5646

96. Han, M.; Kane, R.; Goto, M.; Belfort, G. Discriminate surface molecular recognition sites on a microporous substrate: a new approach. Macromolecules 2003, 36, 4472-4477.

97. Kim, H.; Cohen, R.E.; Hammond, P.T.; Irvine, D.J. Live lymphocyte arrays for biosensing. Adv. Funct. Mater. 2006, 16, 1313-1323.

98. Wang, C.; Ma, Z.; Wang, T.; Su, Z. Synthesis, assembly, and biofunctionalization of silica-coated gold nanorods for colorimetric Biosensing, Adv. Funct. Mater. 2006, 16, 1673-1678

99. Lee, J.; Hernandez,P.; Lee, J.; Govorov, A.O.; Kotov, N.A. Exciton-plasmon interactions inmolecular spring assemblies of nanowires and wavelength-based protein detection. Nature Mater. 2007, 6, 291-295.

100. Ariga, K.; Nakanishi, T.; Hill, J.P.; Shirai, M.; Okuno, M.; Abe, T.; Kikuchi, J. Tunable pK of amino acid residues at the air-water interface gives an L-zyme (Langmuir enzyme). J. Am. Chem. Soc. 2005, 127, 12074-12080.

101. Nakanishi, T.; Miyashita, N.; Michinobu, T.; Wakayama, Y.; Tsuruoka, T.; Ariga, K.; Kurth, D. G. Perfectly straight nanowires of fullerenes bearing long alkyl-chains on graphite. J. Am. Chem. Soc. 2006, 128, 6328-6329.

102. Hill, J.P.; Wakayama, Y.; Schmitt, W.; Tsuruoka, T.; Nakanishi, T.; Zandler, M.L.; McCarty, A.L.; D'Souza, F.; Milgrom, L.R.; Ariga, K. Regulating the stability of 2D crystal structures using an oxidation state-dependent molecular conformation. Chem. Commun., 2006, 2320-2322.

103. Hill, J.P.; Wakayama, Y.; Ariga, K. How molecules accommodate a 2D crystal lattice mismatch: an unusual 'mixed' conformation of tetraphenylporphyrin. Phys. Chem. Chem. Phys. 2006, 8, 5034-5037.

104. Vinu, A.; Hossain, K.Z.; Ariga, K. Recent advances in functionalization of mesoporous silica. $J$. Nanosci. Nanotechnol. 2005, 5, 347-371.

105. Vinu, A.; Mori, T.; Ariga, K. New families of mesoporous materials. Sci. Technol. Adv. Mater., 2006, 7, 753-771.

(C) 2007 by MDPI (http://www.mdpi.org). Reproduction is permitted for noncommercial purposes. 